\title{
BMJ Open Nosocomial infection prevalence in patients undergoing extracorporeal membrane oxygenation (ECMO): protocol for a point prevalence study across Australia and New Zealand
}

\author{
Amanda Corley, ${ }^{1}$ India Lye, ${ }^{1}$ Jayshree D Lavana, ${ }^{2}$ Abhilasha Ahuja, ${ }^{2}$ \\ Chris M Anstey, ${ }^{3,4,5}$ Paul Jarrett, ${ }^{1}$ Emma Haisz, ${ }^{6}$ Rachael Parke, ${ }^{7}$ \\ Vincent Pellegrino, ${ }^{8}$ Hergen Buscher, ${ }^{9}$ John F Fraser, ${ }^{1,2}$ On behalf of the ECMO PP \\ Study Investigators
}

To cite: Corley A, Lye I, Lavana JD, et al. Nosocomial infection prevalence in patients undergoing extracorporeal membrane oxygenation (ECM0): protocol for a point prevalence study across Australia and New Zealand. BMJ Open 2019;9:e029293. doi:10.1136/ bmjopen-2019-029293

\section{- Prepublication history for} this paper is available online. To view please visit the journal (http://dx.doi.org/10.1136/ bmjopen-2019-029293).

Received 21 January 2019 Revised 11 June 2019 Accepted 13 June 2019

\section{Check for updates}

(c) Author(s) (or their employer(s)) 2019. Re-use permitted under CC BY-NC. No commercial re-use. See rights and permissions. Published by BMJ.

For numbered affiliations see end of article.

\section{Correspondence to} Ms Amanda Corley; amanda.corley@health.qld. gov.au

\section{ABSTRACT}

Introduction Extracorporeal membrane oxygenation (ECMO) provides cardiac and/or respiratory support when other therapies fail. Nosocomial infection is reported in up to $64 \%$ of patients receiving ECM0 and increases morbidity and mortality. These patients are at high risk of infection due, in part, to the multiple invasive devices required in their management, the largest being the cannulae through which ECMO is delivered. Prevalence of nosocomial infection in ECMO patients, including ECMO cannula-related infection, is not well described across Australia and New Zealand.

Methods and analysis This is a prospective, observational point prevalence study of 12 months duration conducted at 11 ECM0 centres across Australia and New Zealand. Data will be collected for every patient receiving ECM0 during 12 predetermined data collection weeks. The primary outcome is the prevalence of laboratory-confirmed bloodstream infection, and suspected or probable nosocomial infections; and the secondary outcomes include describing ECMO cannula dressing and securement practices, and adherence to local dressing and securement guidelines. Data collection will be finalised by March 2019.

Ethics and dissemination Relevant ethical and governance approvals have been received. Study results will describe the prevalence of suspected and confirmed nosocomial infection in adult, paediatric and neonatal patients receiving ECMO across Australia and New Zealand. It is expected that the results will be hypothesis generating and lead to interventional trials aimed at reducing the high infection rates seen in this cohort. Results will be published in peer-reviewed journals and presented at relevant conferences.

Trial registration number ANZCTRN12618001109291; Pre-results.

\section{INTRODUCTION}

Extracorporeal ation (ECMO) membrane provides oxygentemporary

\section{Strengths and limitations of this study}

- Patients receiving extracorporeal membrane oxygenation (ECM0) experience high rates of nosocomial infection which increase morbidity and mortality but prevalence is not known across Australia and New Zealand.

- The point prevalence design, incorporating monthly data collection weeks over a 12-month period, will increase sample size of this uncommon treatment, and allow smaller-volume centres to contribute.

- The study design precludes estimates of nosocomial infection incidence in this population.

- Due to the relatively uncommon nature of ECMO, there is a possibility that some patients receiving ECMO will not be captured.

- Results are expected to be hypothesis generating and will lead to targeted interventional trials to reduce infection rates.

cardiorespiratory support to critically ill patients when maximal conventional support has failed. ${ }^{1}$ While ECMO offers patients a chance of overcoming critical illness, it is not without significant risks. ${ }^{1}$ Nosocomial infection is one of the most frequent and serious complications of $\mathrm{ECMO}^{2}{ }^{3}$ and is reported in up to $64 \%$ of patients. ${ }^{4}$ Infections acquired on ECMO are associated with longer periods of mechanical ventilation and ECMO support, ${ }^{2-8}$ as well as longer hospital stays $^{489}$ and increased mortality in the paediatric population. ${ }^{10}$

Factors associated with increased infection risk include adult patients, ${ }^{2}$ severity of underlying illness, ${ }^{49}$ immunosuppression, ${ }^{11}$ longer duration of ECMO support ${ }^{246712}$ and intensive care unit (ICU) stay, ${ }^{6}$ and treatment with 
Veno-arterial (VA) ECMO. ${ }^{211} 12$ The presence of intravascular devices, such as large-bore ECMO cannulae, central venous catheters and intra-arterial lines, could similarly place patients at increased risk of nosocomial infections due to disruption to the skin's protective barrier in addition to potential contamination of the multiple ports of entry on these devices. ${ }^{12}$ ECMO cannula-related infections are difficult to diagnose, due to the inability to routinely replace and culture ECMO cannulae ${ }^{13}{ }^{14}$; and the systemic inflammatory response to the extracorporeal circuit itself. ${ }^{15}$ As a result, ECMO cannula-related infections are likely to be under-diagnosed. Indeed, Messika and colleagues ${ }^{16}$ suggest that primary bloodstream infection (BSI) with an unidentifiable origin should be considered attributable to ECMO cannulae, particularly in the setting of prolonged ECMO treatment.

Data from single-centre studies report ECMO cannula-related infections in 7\%-18\% of patients undergoing $\mathrm{ECMO},{ }^{8} 111317$ with an estimated incidence between 4.8 and 17.2 episodes per 1000 ECMO days. ${ }^{413} 17$ Given the high incidence of ECMO cannula-related infection and the difficulty in reliably diagnosing it, ${ }^{17} 18$ infection prevention measures are vital. Such measures would include the effective dressing and securement of ECMO cannula to prevent micromotion or pistoning of the cannula and subsequent extrinsic contamination of the insertion point. Extensive research has been undertaken to reduce infection in other intravascular devices through effective dressing and securement, ${ }^{19-23}$ but has thus far not occurred in ECMO cannulae. These cannulae are the largest of all intravascular devices with quadruple the rate of infection compared with other vascular devices ( $4.8 \mathrm{vs}$ 1.2 episodes/1000 ECMO days, respectively) ${ }^{17}$ and therefore this must be seen as a research priority.

Before targeted interventions are developed and tested to address nosocomial infection, the prevalence of such infections must be properly estimated. Therefore, we propose a point prevalence study across Australia and New Zealand to identify the prevalence of suspected or confirmed nosocomial infection in adult, paediatric and neonatal ECMO patients, with a particular focus on ECMO cannula-related infection; and to investigate clinical practice regarding dressing and securement of ECMO cannula.

\section{METHODS AND ANALYSIS \\ Study design}

This project is a prospective, observational point prevalence study and will be conducted in 11 ECMO centres across Australia and New Zealand over a 12-month period. Data will be collected for every ECMO patient meeting study inclusion criteria during 12 prespecified 7 day data collection periods, each separated by $\sim 3-4$ weeks. This point prevalence design allows relatively uncommon therapies, such as ECMO, to be captured, while also allowing lower volume ECMO centres the opportunity to participate.

\section{Patient and public involvement}

As this study is an initial exploratory study to collect baseline data, there has been no patient or public involvement in the development of this study protocol.

\section{Participants}

Adult, paediatric and neonatal patients will be included in this study. No formal sample size calculations were completed for this study due to the exploratory nature of the study design. It is anticipated that each participating centre will recruit at least 10 patients over the 12-month recruitment period, giving an expected overall cohort of 120 patients. Inclusion criteria are (1) patients undergoing ECMO treatment with a circuit that includes a membrane oxygenator and pump and (2) patients decannulated from ECMO treatment within the last 48 hours. Exclusion criteria are (1) patients who have had data captured in a previous data collection period, (2) patients decannulated from ECMO $>48$ hours and (3) patients with extracorporeal support that does not include a membrane oxygenator and/or pump. As this study aims to identify the prevalence of hospital-acquired infection, patients will not be required to be free from infection at time of study enrolment.

\section{Outcomes}

\section{Primary outcomes}

1. Laboratory-confirmed BSI, defined $\mathrm{as}^{24}$ :

a. At least one positive culture with an identifiable pathogen/organism; OR

b. Patient has one of the following signs or symptoms: fever $\left(>38.0^{\circ} \mathrm{C}\right)$, chills or hypotension and at least two positive blood cultures for a common skin contaminant within 48 hours (includes coagulase-negative staphylococci, Micrococcus spp, Propionibacterium acnes, Bacillus spp and Corynebacterium spp).

2. Suspected/probable nosocomial infection, defined as: a. BSI:

- Two positive blood cultures for a common skin contaminant from two separate blood samples within the preceding 48 hours (includes coagulase-negative staphylococci, Micrococcus spp, $P$. acnes, Bacillus spp and Corynebacterium spp) without signs or symptoms of infection; OR

- Clinical signs of fever $\left(>38.0^{\circ} \mathrm{C}\right)$, chills, hypotension without positive blood culture and no other identifiable secondary source of infection.

b. Ventilator-associated pneumonia $(\mathrm{VAP})^{25}$ :

- New onset purulent secretions or change in sputum colour, odour, quantity or consistency; AND/ OR

- Radiological evidence of new and persistent infiltrates (two or more serial chest X-rays or CT scans with suggestive images of pneumonia), and two of the following with no other recognised source.

- Fever $>38.0^{\circ} \mathrm{C}$, white cell count $<4.0$ or $>12.0 \times 10^{9} / \mathrm{L}$, new or increasing vasopressor 
requirement, increasing ECMO respiratory support suggestive of worsening gas exchange.

Given the lack of consensus on definition of VAP and the complexities of diagnosing the infection in a patient on ECMO, where clinical, biochemical and radiological signs may be confounded due to the extracorporeal circuit, a combination of clinical and laboratory parameters will be used to identify cases of VAP.

a. Urinary tract infection (UTI) ${ }^{24}$ :

- Two of the following with no other recognised cause:

- Fever $>38.0^{\circ} \mathrm{C}$, urgency, frequency, dysuria, suprapubic tenderness.

- AND at least one of the following:

- Positive dipstick for leucocyte esterase and/or nitrate, pyuria urine specimen with $>10$ White Blood Cell (WBC) $/ \mathrm{mL}$, organisms seen on Gram stain of unspun urine, at least two urine cultures with repeated isolation of the same uropathogen (Gram-negative bacteria or Staphylococcus saprophyticus) with $\geq 102$ colonies $/ \mathrm{mL}$ in unvoided specimens, $\leq 10^{5}$ colonies $/ \mathrm{mL}$ of a single uropathogen (Gram-negative bacteria or S. saprophyticus) when the patient is being treated with effective antimicrobial agents for a UTI.

b. Surgical site infection (SSI) ${ }^{25}$-including intravascular device (inclusive of ECMO cannula) localised infection:

- Infection within 30 days after operation or ECMO cannula or intravascular device insertion and involves incisional skin and subcutaneous tissue and at least one of the following:

- Purulent drainage with or without laboratory confirmation from the incision/insertion site.

- Organisms isolated from an aseptically obtained fluid or tissue culture from the incision/insertion site.

- At least one of the signs or symptoms of infection: pain/tenderness, localised swelling, redness or heat.

- Diagnosis of superficial incisional SSI or intravascular device insertion site made by surgeon or treating intensivist.

\section{Special considerations for neonates}

To avoid misdiagnosis, in addition to the above criteria other clinical signs that would be considered relevant are:

- Fever $\left(>38^{\circ} \mathrm{C}\right.$ ) or temperature instability (frequent post-set of the incubator) or hypothermia $\left(<36.5^{\circ} \mathrm{C}\right)$.

- Tachycardia ( $>200$ beats/min) or new/increased bradycardia ( $<80$ beats $/ \mathrm{min})$.

- Capillary refilling time $>2 \mathrm{~s}$.

- New or increased apnoea(s) (>20s).

- Unexplained metabolic acidosis.

- New-onset hyperglycaemia (>140 mg/dL).

- Unstable general condition of the patient.

- Apathy.

- Increased oxygen requirement.

Relevant laboratory parameters will include:
- Raised C reactive protein $(>20 \mathrm{mg} / \mathrm{L})$.

- Immature/total neutrophil ratio $>0.2$.

- Leucocytes $<5 \times 10^{9} / \mathrm{L}$.

- Platelets $<100 \times 10^{9} / \mathrm{L}^{24}$

\section{Secondary outcomes}

1. ECMO cannula dressing and securement practices for each cannula on the point prevalence day, specifically:

a. Insertion site dressing type:

- Gauze and tape.

- Semipermeable transparent dressing.

- Transparent, chlorhexidine gluconate-impregnated dressing.

- Chlorhexidine gluconate-impregnated disk/ sponge covered with transparent dressing.

- Silver-impregnated dressing.

- Other.

b. Length of time dressing in situ on study day.

c. Sutures to secure cannula at the insertion site and/ or along circuit tubing length.

d. Circuit tubing securement methods:

- Sutures.

- Commercial suture-less fixation device.

- Adhesive bandage or tape.

- Clipping or taping to a fixed object.

- Other

e. Number of circuit tubing fixation points used for each cannula on the point prevalence study day:

- 1 point.

- 2 points.

$->2$ points.

- Other.

2. Presence of local dressing and securement guidelines and compliance of reported cannula dressing and securement methods with these guidelines.

\section{Data collection}

On each study week, trained investigators will screen all ECMO patients for eligibility against the predefined inclusion and exclusion criteria. Using the online database provider Research Electronic Data Capture (REDCap; project-redcap.org), investigators will complete one study-specific electronic case report form per eligible ECMO patient on 1 day during the study week. The data collection tool was pilot tested at the coordinating site prior to use in the larger point prevalence study. Data collected will include patient demographic data; ICU admission and treatment information; ECMO indication, modality and settings; ECMO cannula insertion, dressing and securement data; presence of suspected or confirmed nosocomial infection; and antibiotic therapy. Laboratory samples will be collected and analysed as per local hospital policy, all of which are in accordance with local pathology regulatory bodies.

Each site will keep a recruitment $\log$ of all patients screened and studied, and each recruited patient will be entered on REDCap using a unique study number identifier. Recruitment logs will be stored securely by 
investigators at each site for the purposes of data accountability; will be retained for the appropriate period as per institutional policy; and will not be submitted to the coordinating site. To ensure collected data are consistent across sites, a data dictionary (either adult or paediatric/ neonates) will be supplied to study investigators. Additionally, investigators at each site will receive education from the coordinating site regarding data collection methods and how to remit the data securely.

\section{Statistical analysis}

Data will be checked for discrepancies by a study investigator at the coordinating site, and any queries resolved prior to commencement of data analysis. Statistical analysis will be performed using a proprietary statistical package (STATA V.15.x). Data will be examined in accordance with its type and distribution, with, in the first instance, the use of general data descriptive methods. Mean (SD) will be used for normally distributed data, median (IQR) for non-normal data and proportion (\%) for categorical and binary data. To determine prevalence of infection, each positive patient episode will be counted as one infection. Data regarding multiple infections and/ or organisms will be collected and presented descriptively. Comparative statistics on non-paired data will be performed using tests appropriate for the type of data. That is, the Student's t-test will be used for normal data, the Wilcoxon rank sum test for non-normal data and $\chi^{2}$ and Fisher's exact test for categorical/binary data.

Appropriateness and use of inferential statistical analyses will depend on the final sample size of the cohorts. Initially and where appropriate, detailed inferential analysis will be done using univariate regression techniques. More complex multivariable models will be constructed based on those initial results. Specifically, dichotomous outcome data will be analysed using logistic regression while categorical or continuous data will be analysed using linear regression. Regression diagnostics will be employed to test the adequacy and appropriateness of each model. When present, time to event data will be analysed using a Cox proportional hazards model. The level of significance will be set at $\mathrm{p}<0.05$ unless otherwise dictated by the analytical techniques, for example, when several statistical tests are performed on the same dataset. In this case, a suitable adjustment in $\mathrm{p}$ value will be made (eg, Bonferroni correction).

\section{ETHICS AND DISSEMINATION OF RESULTS}

Low risk ethical approval and a waiver of consent has been provided by the Metro South Hospital and Health Service Human Research Ethics Committee (HREC/17/ $\mathrm{QPAH} / 383)$ for Australian sites. Appropriate site-specific approvals were obtained by all Australian sites. For New Zealand sites, ethics approval was obtained from the Southern Health and Disability Ethics Committee (17/ STH/93). Study findings will be presented at national and international assemblies. Results will be published in peer-reviewed journals in accordance with the Strengthening the Reporting of Observational Studies in Epidemiology Statement requirements.

\section{TRIAL STATUS}

Data collection is ongoing over the 12-month study period and will be complete by March 2019.

\section{DISCUSSION}

Patients undergoing treatment with ECMO experience high rates of nosocomial infection ${ }^{235}$ which is associated with increases in mortality, ${ }^{10}$ duration of ECMO support ${ }^{10}$ and mechanical ventilation, ${ }^{24-7}$ and ICU and hospital lengths of stay. ${ }^{49}$ This multicentre point prevalence study will provide useful data regarding the prevalence of nosocomial infection, including cannula-related local and bloodstream infections, across Australian and New Zealand ECMO populations. Additionally, it will describe ECMO cannula dressing and securement practices across the region. The results will inform future research initiatives aimed at reducing nosocomial infection rates, for instance, a planned randomised controlled trial investigating the most effective method of dressing and securing peripheral ECMO cannulae to reduce infection and cannula dislodgement. Designing strategies to reduce infection rates in this population must be seen as a clinical and research priority, so that patient outcomes can be optimised and healthcare costs reduced.

\section{Author affiliations}

${ }^{1}$ Critical Care Research Group, The Prince Charles Hospital and University of Queensland, Brisbane, Queensland, Australia

${ }^{2}$ Adult Intensive Care Services, The Prince Charles Hospital, Brisbane, Queensland, Australia

${ }^{3}$ Intensive Care Unit, Sunshine Coast University Hospital, Birtinya, Queensland, Australia

${ }^{4}$ School of Medicine, University of Queensland, Brisbane, Queensland, Australia ${ }^{5}$ School of Medicine, Griffith University, Birtinya, Queensland, Australia

${ }^{6}$ Paediatric Intensive Care Unit, Queensland Children's Hospital, South Brisbane, Queensland, Australia

${ }^{7}$ Cardiothoracic and Vascular Intensive Care Unit, Auckland City Hospital, Auckland, New Zealand

${ }^{8}$ Department of Intensive Care, Alfred Hospital, Melbourne, Victoria, Australia ${ }^{9}$ Department of Intensive Care Medicine, St Vincent's Hospital, Sydney, New South Wales, Australia

Acknowledgements The authors would like to thank all the contributors to this study, without whom the study will not be possible: Adult Intensive Care Services, The Prince Charles Hospital, Brisbane. Queensland Children's Hospital, Brisbane: Luregn Schlapbach; The Princess Alexandra Hospital, Brisbane: Jason Meyer, James Walsham; Gold Coast University Hospital, Gold Coast: Mandy Tallott, James Winearls; Westmead Hospital, Sydney: Louise Cope, Adam Hastings; The Children's Hospital at Westmead, Sydney: Amelia Griffiths, Marino Festa; St Vincent's Hospital, Sydney: Claire Reynolds; The Alfred Hospital, Melbourne: Emma Martin, Shirley Valance, Jayne Sheldrake; The Royal Children's Hospital, Melbourne: Derek Best, Roberto Chiletti; Auckland City Hospital, Auckland: Eileen Gilder, Magdalena Butler, Keri-Anne Cowdrey, Jane Hallion, Philippa Neal; Starship Children's Hospital, Auckland: Claire Sherring, Miriam Rea, Nicola Gini. The authors would like to also acknowledge the National Health and Medical Research Council funded Centre of Research Excellence in Advanced Cardio-respiratory Therapies Improving OrgaN Support (CRE ACTIONS). 
Contributors AC conceived the study, wrote the grant proposal, developed the protocol, wrote the manuscript and approved the final draft. IL contributed to the grant proposal and protocol development, prepared and coordinated ethical and governance applications, performed data collection and coordination, reviewed the manuscript and approved the final draft. JDL, AA, VP and HB contributed to protocol development, reviewed the manuscript and approved the final draft. CMA contributed to protocol development, provided statistical advice and data analysis plan, reviewed the manuscript and approved the final draft. PJ contributed to protocol development, data collection methods, reviewed the manuscript and approved the final draft. EH and RP contributed to protocol development, reviewed the manuscript and approved the final draft. JFF contributed to grant proposal, protocol development, reviewed the manuscript and approved the final draft.

Funding This study received funding from The Prince Charles Hospital Foundation (IACB2017-01).

Competing interests None declared.

Patient consent for publication Not required.

Provenance and peer review Not commissioned; externally peer reviewed.

Open access This is an open access article distributed in accordance with the Creative Commons Attribution Non Commercial (CC BY-NC 4.0) license, which permits others to distribute, remix, adapt, build upon this work non-commercially, and license their derivative works on different terms, provided the original work is properly cited, appropriate credit is given, any changes made indicated, and the use is non-commercial. See: http://creativecommons.org/licenses/by-nc/4.0/.

\section{REFERENCES}

1. Combes A, Brodie D, Chen YS, et al. The ICM research agenda on extracorporeal life support. Intensive Care Med 2017;43:1306-18.

2. Bizzarro MJ, Conrad SA, Kaufman DA, et al. Extracorporeal Life Support Organization Task Force on Infections ECMO. Infections acquired during extracorporeal membrane oxygenation in neonates, children, and adults. Pediatr Crit Care Med 2011;12:277-81.

3. Biffi S, Di Bella S, Scaravilli V, et al. Infections during extracorporeal membrane oxygenation: epidemiology, risk factors, pathogenesis and prevention. Int J Antimicrob Agents 2017;50:9-16.

4. Schmidt M, Bréchot N, Hariri S, et al. Nosocomial infections in adult cardiogenic shock patients supported by venoarterial extracorporeal membrane oxygenation. Clin Infect Dis 2012;55:1633-41.

5. Burket JS, Bartlett RH, Vander Hyde K, et al. Nosocomial infections in adult patients undergoing extracorporeal membrane oxygenation. Clin Infect Dis 1999;28:828-33.

6. Hsu MS, Chiu KM, Huang YT, et al. Risk factors for nosocomial infection during extracorporeal membrane oxygenation. $J$ Hosp Infect 2009;73:210-6.

7. Sun HY, Ko WJ, Tsai PR, et al. Infections occurring during extracorporeal membrane oxygenation use in adult patients. $J$ Thorac Cardiovasc Surg 2010;140:1125-32.

8. Davies A, Jones D, Bailey M, et al. Extracorporeal Membrane Oxygenation for 2009 Influenza A(H1N1) Acute Respiratory Distress Syndrome. JAMA 2009;302:1888-95.
9. Aubron C, Cheng AC, Pilcher D, et al. Infections acquired by adults who receive extracorporeal membrane oxygenation: risk factors and outcome. Infect Control Hosp Epidemiol 2013;34:24-30.

10. Montgomery VL, Strotman JM, Ross MP. Impact of multiple organ system dysfunction and nosocomial infections on survival of children treated with extracorporeal membrane oxygenation after heart surgery. Crit Care Med 2000;28:526-31.

11. Austin DE, Kerr SJ, Al-Soufi S, et al. Nosocomial infections acquired by patients treated with extracorporeal membrane oxygenation. Crit Care Resusc 2017;19(Suppl 1):68-75.

12. O'Neill JM, Schutze GE, Heulitt MJ, et al. Nosocomial infections during extracorporeal membrane oxygenation. Intensive Care Med 2001;27:1247-53.

13. Allou N, Lo Pinto H, Persichini R, et al. Cannula-Related Infection in Patients Supported by Peripheral ECMO: Clinical and Microbiological Characteristics. Asaio J 2019;65:180-186.

14. Hahne K, Horstmann C, Fischer D, et al. Cannula-related infection in adult medical intensive care unit patients undergoing extracorporeal life support and extracorporeal membrane oxygenation. $J$ Hosp Infect 2015;91:372-4.

15. Millar JE, Fanning JP, McDonald $\mathrm{Cl}$, et al. The inflammatory response to extracorporeal membrane oxygenation (ECMO): a review of the pathophysiology. Crit Care 2016;20:387.

16. Messika J, Schmidt M, Aubry A, et al. Extracorporeal Membrane Oxygenation-Associated Infections: Carefully Consider Cannula Infections!. Crit Care Med 2018;46:e171-e2.

17. Thomas G, Hraiech S, Cassir N, et al. Venovenous extracorporeal membrane oxygenation devices-related colonisations and infections. Ann Intensive Care 2017;7:111.

18. ELSO Infectious Diseases Taskforce. Infection Control and Extracorporeal Life Support. Ann Arbor, MI, USA 2014.

19. Maki DG, Stolz SS, Wheeler S, et al. A prospective, randomized trial of gauze and two polyurethane dressings for site care of pulmonary artery catheters: implications for catheter management. Crit Care Med 1994;22:1729-37.

20. Rickard CM, Marsh N, Webster J, et al. Dressings and securements for the prevention of peripheral intravenous catheter failure in adults (SAVE): a pragmatic, randomised controlled, superiority trial. Lancet 2018;392:419-30.

21. Günther SC, Schwebel C, Hamidfar-Roy R, et al. Complications of intravascular catheters in ICU: definitions, incidence and severity. A randomized controlled trial comparing usual transparent dressings versus new-generation dressings (the ADVANCED study). Intensive Care Med 2016;42:1753-65.

22. Ullman AJ, Kleidon T, Gibson V, et al. Innovative dressing and securement of tunneled central venous access devices in pediatrics: a pilot randomized controlled trial. BMC Cancer 2017;17:595.

23. Timsit JF, Mimoz O, Mourvillier B, et al. Randomized controlled trial of chlorhexidine dressing and highly adhesive dressing for preventing catheter-related infections in critically ill adults. Am J Respir Crit Care Med 2012;186:1272-8.

24. European Centre for Disease Prevention and Control. Point prevalence survey of healthcare-associated infections and antimicrobial use in European acute care hospitals - protocol version 5.3. Stockholm: ECDC 2016.

25. Centers of Disease Control and Prevention. National Healthcare Safety Network (NHSN) Patient Safety Component Manual. Washington, DC: Centers of Disease Control and Prevention, 2017. 